\title{
Physical activity and mental health in women with Polycystic Ovary Syndrome
}

\author{
Lauren K Banting ${ }^{1}$, Melanie Gibson-Helm², Remco Polman ${ }^{1,3}$, Helena J Teede ${ }^{2,4}$ and Nigel K Stepto ${ }^{1,2,3^{*}}$
}

\begin{abstract}
Background: Physical activity is prescribed as a component of primary management for Polycystic Ovary Syndrome (PCOS). This study investigates the association between physical activity and mental health as well as the exercise barriers, motivators and support providers for younger women with and without PCOS to assist in physical activity uptake and prescription for these women.

Methods: Women aged $18-50$ years with $(n=153)$ and without PCOS $(n=64)$ completed a questionnaire at one time point. The questionnaire included the Hospital Anxiety and Depression Scale and a survey regarding levels of physical activity, physical activity barriers, motivators and supports. A MANCOVA assessed associations between physical activity, PCOS and mental health (specifically depression and anxiety). Descriptive and Chi square goodness of fit statistics assessed the differences in perceived barriers, motivators and support providers amongst women with and without PCOS.
\end{abstract}

Results: Women with PCOS displayed higher severity of depression $(F(1,210)=8.32, p=0.004)$ and anxiety $(F(1,210)=$ $17.37, p<0.001)$ symptoms compared to controls. Overall, for physically active women, depression was significantly less severe than in their inactive counterparts $(F(2,210)=13.62, p<0.001)$. There were no differences in anxiety by physical activity status and no interaction effects between PCOS and activity status for depression or anxiety. Women with PCOS were more likely to report a lack of confidence about maintaining physical activity $\left(X^{2}=3.65 ; p=0.046\right)$, fear of injury $\left(X^{2}=4.08 ; p=0.043\right)$ and physical limitations $\left(X^{2}=11.92 ; p=0.001\right)$ as barriers to physical activity and were more likely to be motivated to be active to control a medical condition $\left(X^{2}=7.48 ; p=0.006\right)$. Women with PCOS identified more sources of support compared to women without PCOS.

Conclusions: Physical activity is associated with lower depression in women with PCOS and differences exist in the self-reported physical activity barriers, motivators and support providers, compared to controls. Being more active may offer mental health benefits in managing PCOS. Prescribing physical activity to women with PCOS should be individualized and consider both common and PCOS-specific barriers and motivators for successful engagement.

Keywords: Depression, Anxiety, Exercise, Motivation, Chronic disease, PCOS, Polycystic ovary syndrome

\section{Background}

Polycystic ovary syndrome (PCOS) is one of the most common and complex endocrine disorders and the leading cause of anovulatory infertility in reproductive aged women. PCOS affects between $12-21 \%$ of reproductive aged women, depending on diagnostic criteria, with many

\footnotetext{
* Correspondence: nigel.stepto@vu.edu.au

${ }^{1}$ Institute of Sport Exercise and Active Living, Victoria University, Melbourne, Victoria, Australia

Women's Public Health Research, Monash Centre for Health Research and Implenetation, School of Public Health and Preventive Medicine, Monash University, Clayton, Victoria, Australia

Full list of author information is available at the end of the article
}

cases being undiagnosed $[1,2]$. PCOS has reproductive, psychological and cardio-metabolic features [3-7] and is associated with many long term adverse health problems including increased risk of obesity $[4,8,9]$, type 2 diabetes and metabolic impairments [10-13], and cardiovascular risk factors $[14,15]$. We and others have shown that diminished mental health is related to PCOS, including depression, anxiety and lower quality of life [16-18]. These mental health differences are noted across the lifespan, including adolescents, and across the different PCOS phenotypes $[19,20]$.

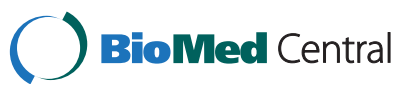


Many chronic illnesses have mental health impacts and are associated with reduction in quality of life and increases in depressive symptoms [21-24]. In PCOS, symptoms and co-morbidities increase the risk of adverse mental health consequences. Coping with the condition itself, fears regarding infertility, loss of femininity and sexuality, body image concerns and lower self-worth may all contribute to poorer mental health outcomes [7,18,25]. Mental health is especially relevant in PCOS management as it is vital to self-efficacy around a healthy lifestyle (including physical activity). Therefore optimisation of physical activity as a treatment for PCOS, as recommended by the first Evidence based Guideline for the Assessment and Management of PCOS [26], should consider the mental health status of women with PCOS and the interactions with physical activity.

Lifestyle management, including healthy diet and physical activity, is currently advised as the first line management strategy for PCOS [26]. Physical activity is an effective therapeutic option for the reproductive $[27,28]$ and metabolic features [29] of PCOS. The specific interaction between physical activity and mental health has not been explored in depth in PCOS, however, preliminary data including a recent clinic-based study of women with PCOS found physically inactive women had higher depression scores than physically active women, and there was an association between lower physical activity and mild depression [30]. Reductions in depression and elevations in quality of life have been observed in combined physical activity and dietary interventions in PCOS [31] and specific physical activity interventions have been found to be effective in reducing mild-moderate depression [32,33] and anxiety [34] in the general population. Yet engagement and sustainability in physical activity in women with PCOS is particularly problematic. Despite the positive physical effects of exercise [35], the inherent symptoms and co-morbidities associated with PCOS may present barriers to engagement in physical activity and may impact on typically positive psychological responses to being physically active. Thus, research investigating the relationship between physical activity and mental health for women with PCOS and exploration of the enablers and the barriers to activity is important for appropriate prescription of physical activity Additionally, as attrition in PCOS physical activity research interventions is typically high (30-40\%) [29,31,35] and physical activity maintenance in PCOS is generally inadequate, understanding the barriers and motivators of physical activity for women with PCOS is required to promote engagement and sustainability. Little is known about the barriers and motivators of physical activity that specifically effect women with PCOS, and being the first study to explore physical activity barriers and motivators among this population of women, the study was considered hypothesis generating.
We hypothesised that physical activity would be associated with better mental health, specifically a lower severity of both anxiety and depression, for all women. Furthermore, the study aimed to describe the common barriers and motivators to physical activity for women with PCOS compared to a control sample of women. We expected some barriers and motivators to differ between women with and without PCOS and that they may vary according to age [36].

\section{Methods \\ Participants}

This sub-study focused on physical activity as part of a broader study investigating health-related behaviour in women with and without PCOS. Study recruitment has been described in detail previously [18]. This was a cross-sectional, observational study utilising devised and validated questionnaires. Southern Health Human Research Ethics Committee C (Project No. 07070C) approved this study and the procedures involved. All women provided informed consent to participate in the study. Women responded to recruitment advertisements across a range of community health settings including medical clinics, print media, online women's health sites and an online PCOS support group. Women needed to be living in Australia, aged between 18 and 70 years and able to read and write in English. Women with and without PCOS were recruited in this manner. PCOS status was then determined based on self-reported previous medical diagnosis (Rotterdam criteria) [37] and the presence of at least two of the following (self-reported during phone screening); polycystic ovaries on ultrasound, high androgen levels/clinical hyperandrogenism and menstrual irregularity. Only the women aged 18- 50 years were included for analysis to reduce any potential confounding effects of menopause (average age of onset is 51 years) which is associated with mental health outcomes [38]. Pregnant women, women living overseas, women with a psychiatric illness (other than anxiety or depression) or an incomplete PCOS diagnosis were excluded from the research. Participants completed the survey in either online or written format according to their preference.

\section{Measures}

\section{Body mass index}

BMI was calculated based on self-reported weight and height (weight/height $\left.(\mathrm{m})^{2}\right)$. This population (adult women) is known to typically underestimate their weight and overestimate their height (therefore reducing estimated BMI) [39], however, any adjustments required would be common to both groups. 


\section{Physical activity}

A simple, devised measure based on the trans-theoretical model TTM; [40] was used to assess participants' physical activity levels. As this was part of a large study exploring many aspects of health related behaviour this tool was used rather than longer physical activity tools or pedometers to reduce the burden on participants while gaining a general indication of the individual's habitual physical activity. A definition of physical activity based on the National Physical Activity Guidelines for Australians was provided to participants. These guidelines define physical activity in a very similar way to other organisations including the World Health Organisation [41]. Participants selected a statement of agreement with each phrase typically used to describe the pre-contemplation (I currently do not exercise and I don't intend to start exercising any time soon), contemplation (I currently do not do exercise however I am thinking about exercising in the next few months), preparation (I occasionally exercise now, but not on a regular basis), action (I currently exercise on a regular basis and have done so for the past 6 months) and maintenance (I currently exercise on a regular basis and have done so for more than 6 months) stages of the TTM. Responses ranged from 1 (Definitely agree) to 4 (Definitely disagree). Women were classified as physically active if they reported agreement (definite or somewhat agreement) for the active or maintenance stages of the TTM questionnaire.

\section{Mental health measures}

The Hospital Anxiety and Depression Scale HADS; [42] is a state measure of anxiety and depression, used to detect clinical cases and the severity of both conditions. The scale consists of 14 items, 7 measuring depression, 7 measuring anxiety. The measure displays high internal consistency for both anxiety $(\alpha=.93)$ and depression $(\alpha=.90)$ [43]. The scores can be used as a continuous variable, with higher scores (out of a possible 21) indicating greater anxiety or depression. A score of 8 or greater indicates possible presence of anxiety and/or depression.

\section{Physical activity barriers and motivators questionnaire}

Women were presented with two lists comprised of commonly reported physical activity barriers and motivators. Participants were asked to nominate which of the barriers were 'main factors that prevent/stop' them from engaging in physical activity. The items included 12 barriers commonly associated with physical activity. Participants could also nominate barriers in the 'other' category. This option resulted in 'no enjoyment' being analysed as a $13^{\text {th }}$ barrier. For the motivators, participants were asked to identify the items which they considered to be 'the main advantages of engaging in physical activity'. Items included 13 motivators frequently cited as reasons for physical activity and again, participants could nominate other items which resulted in 'enjoyment/fun' and 'time to think' being included for analysis. The barriers and motivators were selected from international physical activity literature with specific local barriers and motivators selected from the Main Barriers and Motivators Report by the Australian Bureau of Statistics [44].

\section{Physical activity supports}

From a list of 9, women were asked to select individuals or groups that 'are most important to you when you think about engaging in physical activity'. These supports were chosen from previous literature and current Australian physical activity reports concerning physical activity facilitation [44]. Women could select as many as relevant and nominate in an 'other' category, resulting in 'myself', 'no one', 'exercise acquaintances or team mates' and 'pets' being included for analysis.

\section{Data analysis \\ Statistical analysis}

Analysis was performed using SPSS version 19. The outcome variables, anxiety and depression, were determined to be normally distributed. A two-way between groups MANCOVA was conducted to determine significant mean differences in mental health (anxiety and depression) amongst the sample according to physical activity (active vs. inactive) and PCOS (PCOS vs. non PCOS). Age and BMI were used as covariates given the significant role each variable has been shown to have on mental health $[38,45]$. Bonferroni adjustments were used to assess statistical significance for single dependent variables (two-sided $p$-value of 0.025). Independent $t$-tests were used to assess individual differences according to PCOS and physical activity. Numbers of physically active and inactive women with and without PCOS were assessed using a chi-square goodness of fit analysis with statistical significance assumed with a two-sided $p$ value of 0.05. Barriers, motivators and physical activity supports were assessed using descriptive statistics, calculating percentages of each group reporting the barrier as a main factor that prevents physical activity which were compared using chi-square goodness of fit analyses. Results were assessed across the entire sample and also considering age groups (18-30 years, $31-40$ years and 41-50 years to represent typical life stages) to determine whether barriers, motivators and supports differed. The barriers, motivators and supports were also compared between groups in only healthy weight women $(\mathrm{BMI}=$ 18-25). The number of barriers, motivators and supports identified by each group was compared using an independent samples $t$-test. Statistical significance was assumed with a two-sided $p$-value of 0.05 . 


\section{Results}

\section{Participation and demographics}

After considering the inclusion criteria, 240 women were included for analysis. Twenty three $(9.6 \%)$ women were excluded from further analysis due to inconsistent responses to the TTM questions for physical activity. Women who indicated agreement with both inactive and active stages (or disagreement with all stages) were removed from analysis as this response pattern indicates a misunderstanding of the question, or erratic physical activity behaviour. The remaining sample $(N=217)$ were included in the analysis and had a mean age of 31.84 years $(S D=7.74)$ and were on average overweight with a mean body mass index (BMI) of 29.23 ( $S D=$ 7.90). Women with PCOS accounted for $70.5 \%$ of the sample $(n=153)$ and had a higher mean BMI than the control group (Table 1). Of the 40 women born overseas 17 (43\%) were born in Europe (United Kingdom of Great Britain, Italy, Portugal, Netherlands), 11 (28\%) were born in Asia (Hong Kong, India, Sri Lanka, Indonesia, Laos, Malaysia, Singapore), 5 (12\%) were born in Africa (Kenya, Zimbabwe, South Africa), 5 (12\%) were born in Oceania (New Zealand, Papua New Guinea) and 2 (5\%) were born in America (Chile, United States of America).

Table 1 Descriptive statistics according to PCOS status

\begin{tabular}{|c|c|c|c|}
\hline & $\begin{array}{c}\text { PCOS } \\
n=153\end{array}$ & $\begin{array}{c}\text { Control } \\
n=64\end{array}$ & $P$-value \\
\hline Age (SD) & $31.99(6.80)$ & $31.50(9.69)$ & 0.674 \\
\hline BMI $\left(\mathrm{kg} / \mathrm{m}^{2}\right)$ & $31.32(7.71)$ & $24.15(5.77)$ & $<0.001$ \\
\hline Diagnosed infertility (\%) & 37 & 0 & - \\
\hline Highest level of education & & & 0.897 \\
\hline No formal qualification (\%) & 13 & 17 & \\
\hline Formal qualification & 87 & 83 & \\
\hline Born in Australia (\%) & 88 & 67 & $<0.001$ \\
\hline Children (\% with child) & 43 & 27 & 0.027 \\
\hline Living in urban setting (\%) & 75 & 80 & 0.444 \\
\hline Living arrangements & & & 0.034 \\
\hline Living alone (\%) & 5 & 13 & \\
\hline With partner only (\%) & 31 & 30 & \\
\hline With partner and children (\%) & 37 & 19 & \\
\hline With children only (\%) & 3 & 2 & \\
\hline Other adults (\%) & 24 & 38 & \\
\hline Income & & & 0.178 \\
\hline Below \$59,999 (\%) & 26 & 24 & \\
\hline$\$ 60,000-\$ 79,999(\%)$ & 21 & 17 & \\
\hline$\$ 80,000+(\%)$ & 46 & 48 & \\
\hline Don't know/no answer (\%) & 7 & 11 & \\
\hline
\end{tabular}

\section{Physical activity, anxiety and depression}

The Chi-square goodness of fit analysis indicated that more women without PCOS (64\%) were physically active than women with PCOS $(48 \%)(p=0.028)$. After covarying/accounting for age and BMI, both physical activity level (Wilk's Lambda $=0.93$ ) and PCOS status (Wilk's Lambda $=0.92$ ) were significantly associated with the mental health of the participants (combined dependent variable of anxiety and depression). BMI had a significant independent influence on mental health with higher BMI associated with poorer mental health (Wilks's Lambda $=0$ .94) yet no effect was observed for the covariate of age. Women with PCOS (mean $=9.42[\mathrm{SD}=3.76]$ ) had higher anxiety scores than women without PCOS (6.60 [3.63]). This difference was significant $(F(1,210)=17.37, p<0.001$, partial $\left.\eta^{2}=0.08\right)$. Women with PCOS (5.67 [3.76]) also had higher depression scores than women without PCOS (3.05 [3.15]), a difference which was also significant $(F$ $(1,210)=8.32, p=0.004$, partial $\left.\eta^{2}=0.04\right)$. Activity level was not associated with anxiety $(F(2,210)=1.79, p=0.18$, partial $\eta^{2}=0.01$ ) but was significantly associated with depression $\left(F(2,210)=13.62, \quad p<0.001\right.$, partial $\left.\eta^{2}=0.06\right)$. Physically active women (3.53 [2.88]) had significantly lower depression scores than inactive women (6.41 [4.07]). No significant effects were observed for the interaction of PCOS status and activity level. The descriptive statistics according to group and physical activity are presented in Figure 1.

\section{Exercise barriers}

Table 2 details the exercise barriers identified by women with and without PCOS. The number of exercise barriers amongst women with and without PCOS was similar $(t=$ $1.54, p=0.216$ ) with each group identifying approximately four barriers. The barriers were assessed according to age groups (18-30, 31- 40 and 41-50 years) and were determined to be similar in terms of the five most frequently reported exercise barriers and so results are presented for the whole sample. Lack of time and fatigue were the main exercise barriers for both groups. Women with PCOS were more likely to report a lack of confidence regarding their ability to maintain exercise as a barrier $(71 \%$ of PCOS women, $50 \%$ of control women, $p=0.046)$. This barrier was the third most commonly cited barrier for both groups. Women with PCOS were also more likely to report fear of injury and physical limitations as barriers to exercise compared to the women in the control group.

\section{Exercise motivators}

The motivators identified by women with and without PCOS are presented in Table 3. There were no differences between women in terms of PCOS status for the number of motivators reported. Women with PCOS reported no more motivators $(x=8.51, S D=3.27)$ than 


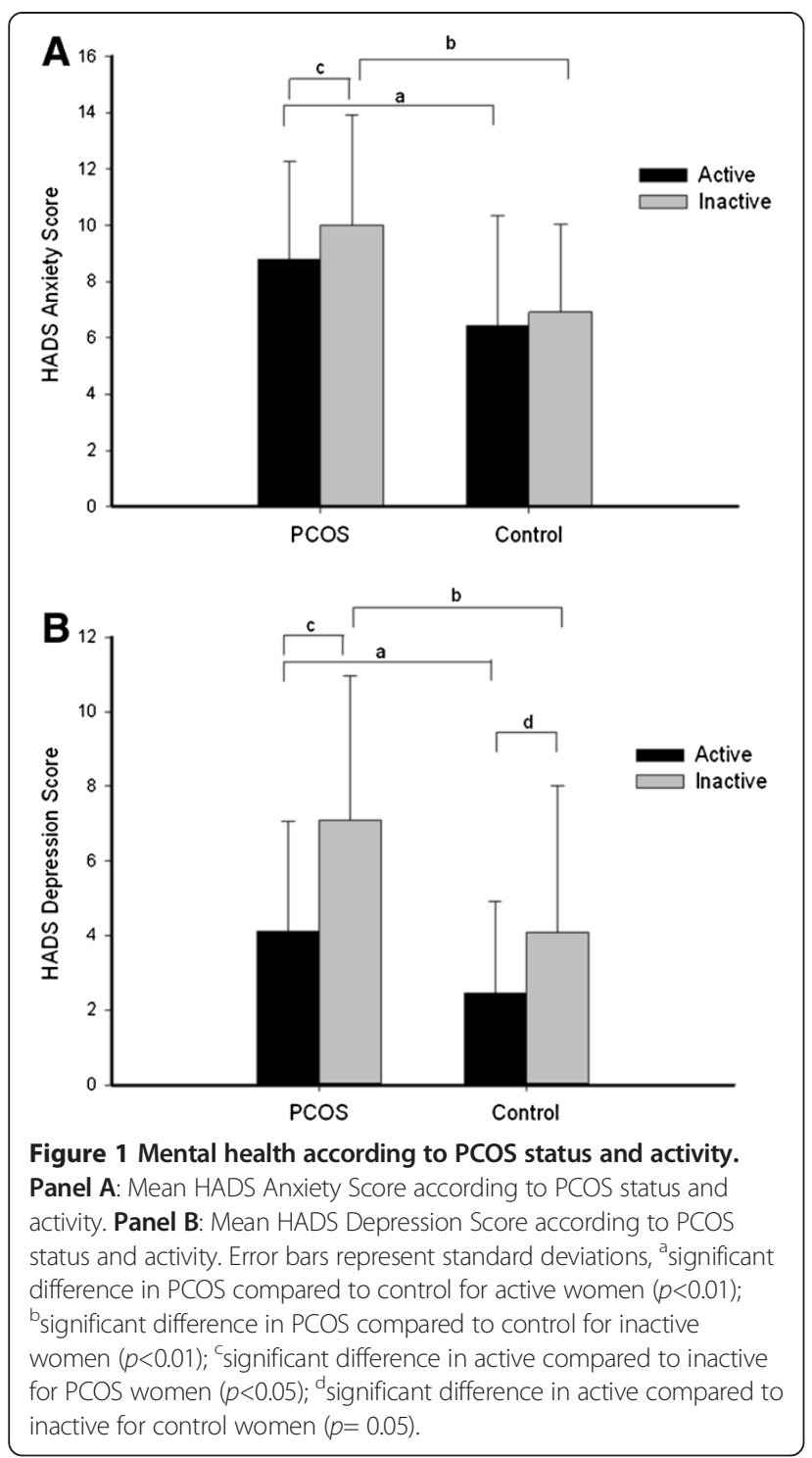

women without PCOS $(x=8.27, S D=2.68)(t=0.27, p=$ $0.607)$. Results are presented for the whole sample after the top five motivators were assessed across age groups and were determined to be similar. Weight control, health improvements, increased energy, stress reduction and health maintenance were the most commonly cited motivators in both groups. Controlling a medical condition $(\mathrm{PCOS}=40 \%$, Control $=19 \%, p=0.006)$ was more commonly cited as an exercise motivator by women with PCOS.

\section{Physical activity supports}

Table 4 summarises the exercise support providers identified by women with and without PCOS. Women with PCOS $(x=3.80, S D=1.96)$ were able to identify approximately 1 more support source than women without PCOS $(\mathrm{x}=3.20, \mathrm{SD}=1.69)(t=4.19, p=0.041)$. The 18 -30 years
Table 2 Barriers to exercise for women with and without PCOS

\begin{tabular}{lccc}
\hline Barrier & PCOS (\%) & Control (\%) & $\boldsymbol{X}^{\mathbf{2}}(\boldsymbol{p})$ \\
\hline Lack of time & $121(79)$ & $46(72)$ & $0.33(0.569)$ \\
Fatigue & $118(77)$ & $44(69)$ & $0.44(0.508)$ \\
Not confident can maintain* & $109(72)$ & $32(50)$ & $3.97(0.046)$ \\
Tiredness & $57(37)$ & $16(25)$ & $2.32(0.128)$ \\
Fear of injury* & $47(31)$ & $11(17)$ & $4.08(.043)$ \\
Physical limitations* & $45(29)$ & $5(8)$ & $11.92(0.001)$ \\
Bad weather & $45(29)$ & $20(31)$ & $0.07(0.796)$ \\
No motivation & $40(26)$ & $24(38)$ & $2.25(0.134)$ \\
Not important & $39(25)$ & $13(20)$ & $0.56(0.456)$ \\
Cost & $26(17)$ & $10(16)$ & $0.03(0.862)$ \\
Young children & $17(11)$ & $4(6)$ & $1.47(0.225)$ \\
Illness & $13(9)$ & $3(5)$ & - \\
Spousal influence & $4(3)$ & $1(2)$ & - \\
Other & $3(2)$ & $0(0)$ & - \\
No enjoyment & $0(0)$ & $0(0)$ & - \\
\hline
\end{tabular}

Note: *indicates significant difference between groups based on Chi-Square goodness of fit analyses $(p<0.05)$.

old age group reported different support groups to the rest of the sample and so results are presented for this group separately (Table 4). In all groups the husband or partner was the primary support provider, followed by friends and parents. The 18-30 years old age group reported physical activity instructors to be one of the top five support providers, whilst children were primary support providers for

Table 3 Exercise motivators for women with and without PCOS

\begin{tabular}{lccc}
\hline Motivator & PCOS (\%) & Control (\%) & $\boldsymbol{X}^{\mathbf{2}}(\boldsymbol{p})$ \\
\hline Control weight & $144(94)$ & $52(81)$ & $0.97(0.326)$ \\
Improve health & $142(93)$ & $49(77)$ & $1.51(0.220)$ \\
Increase energy & $128(84)$ & $47(73)$ & $0.77(0.380)$ \\
Stress reduction & $123(80)$ & $47(73)$ & $0.32(0.571)$ \\
Maintain health & $121(79)$ & $45(70)$ & $0.54(0.461)$ \\
Improve mood & $117(76)$ & $43(67)$ & $0.57(0.452)$ \\
Maintain fitness & $115(75)$ & $51(80)$ & $0.16(0.688)$ \\
Improve self confidence & $103(67)$ & $34(53)$ & $1.63(0.201)$ \\
Decrease risk of disease & $100(65)$ & $37(58)$ & $0.40(0.528)$ \\
Decrease risk of illness & $71(46)$ & $31(48)$ & $0.04(0.837)$ \\
Control medical condition* & $61(40)$ & $12(19)$ & $7.48(0.006)$ \\
Control blood glucose levels & $59(39)$ & $16(25)$ & $3.06(0.080)$ \\
Social interaction & $54(35)$ & $16(25)$ & $1.67(0.197)$ \\
Other & $1(1)$ & $1(2)$ & - \\
Enjoyment & $1(1)$ & $1(2)$ & - \\
Own space & $0(0)$ & $1(2)$ & - \\
\hline
\end{tabular}

Note: *indicates significant difference between groups based on Chi-Square goodness of fit analyses $(p<0.05)$. 
Table 4 Support providers for women with and without PCOS: whole sample

\begin{tabular}{|c|c|c|c|c|c|c|}
\hline \multirow[b]{2}{*}{ Support } & \multicolumn{3}{|c|}{ Whole sample } & \multicolumn{3}{|c|}{ 18- 30 years old sample } \\
\hline & PCOS (\%) & Control (\%) & $X^{2}(p)$ & $\mathrm{PCOS}(\%)$ & Control (\%) & $X^{2}(p c p$ \\
\hline Husband/partner $^{a}$ & $116(76)$ & $37(58)$ & $2.42(0.120)$ & $52(74)$ & $14(42)$ & $8.83(0.003)$ \\
\hline Friends & $93(61)$ & $30(47)$ & $1.82(0.178)$ & $46(66)$ & $22(67)$ & $0.01(0.931)$ \\
\hline Parents & $86(56)$ & $24(38)$ & $3.45(0.063)$ & $49(70)$ & $21(64)$ & $0.27(0.604)$ \\
\hline Other family* & $77(50)$ & $19(30)$ & $5.00(0.025)$ & $42(60)$ & $14(42)$ & $3.18(0.075)$ \\
\hline Children*a & $65(42)$ & $16(25)$ & $4.31(0.038)$ & $15(21)$ & $2(6)$ & $8.33(0.004)$ \\
\hline Health care profs & $42(27)$ & $14(22)$ & $0.51(0.475)$ & $22(31)$ & $8(24)$ & $0.89(0.345)$ \\
\hline Co-workers & $40(26)$ & $13(20)$ & $0.78(0.376)$ & $24(34)$ & $9(27)$ & $0.80(0.370)$ \\
\hline Physical activity instructors & $40(26)$ & $14(22)$ & $0.33(0.564)$ & $25(36)$ & $10(30)$ & $0.55(0.460)$ \\
\hline Myself & $9(6)$ & $4(6)$ & $0.00(1.00)$ & $5(7)$ & $4(12)$ & $1.32(0.251)$ \\
\hline Other & $4(3)$ & $0(0)$ & - & $2(3)$ & $0(0)$ & - \\
\hline Exercise friends/team & $2(1)$ & $2(3)$ & - & 2 (3) & $0(0)$ & - \\
\hline No one & $1(1)$ & $1(2)$ & - & $0(0)$ & 1 (3) & - \\
\hline Pets & $1(1)$ & $1(2)$ & - & $1(1)$ & $0(0)$ & - \\
\hline
\end{tabular}

Note: *indicates significant difference between groups in whole sample based on Chi-Square goodness of fit analyses ( $p<0.05$ ).

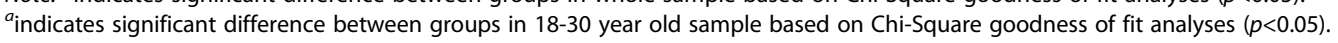

the other age groups. For the whole sample, other family were more frequently cited by women with PCOS as support providers (PCOS $=50 \%$, Control $=30 \%, p=0.025)$, as were children $(\mathrm{PCOS}=43 \%$, Control $=25 \%, p=0.038$ ).

\section{Discussion}

In this novel formative research, fewer women with PCOS reported being physically active compared to women without PCOS. Additionally, women with PCOS had poorer mental health, with higher depression and anxiety scores. Relationships between activity and mental health showed that being physically active was associated with lower depression scores, after accounting for age, BMI and PCOS status. There was no interaction effect of PCOS status and physical activity; depression scores were lower in both physically active groups similarly. Physical activity was not significantly associated with lower anxiety after controlling for age, BMI and PCOS status. Women with PCOS did not identify additional barriers or motivators for physical activity, although they did identify an additional supportive influence than women without PCOS. The barriers, motivators and support providers were similar between groups, yet women with PCOS more often reported a lack of confidence in their ability to maintain exercise, fear of injury and physical limitations as barriers to physical activity. These women were also more likely to report being motivated to do physical activity in order to control a medical condition.

As research and practice regarding physical activity prescription as a management strategy for women with PCOS has been significantly limited by poor engagement and sustainability, formative research is important to assess the association between mental health and activity research to explore the physical activity barriers, motivators and support providers for women with PCOS. The current novel findings from a multiethnic community based sample, show that physical activity was associated with lower depression symptoms compared to physical inactivity for all women after accounting for age, BMI and PCOS. Unsurprisingly, both BMI and PCOS were significantly associated with depression as has been found in previous research $[19,46]$. The current results support findings from a clinic-based study that being physically active is associated with lower rates of depression in women with PCOS [30]. Current results also strengthen claims that participation in physical activity programs may be associated with lower depressive symptoms and improvements in quality of life [31,47] and indicate the value of exploring these associations further using interventional and longitudinal research.

Physical activity was not significantly associated with lower anxiety compared to being inactive after accounting for age, BMI and PCOS. A higher BMI was associated with greater anxiety which is consistent with previous research [46]. Having PCOS was also associated with higher anxiety, supporting the claim that PCOS is associated with poor mental health outcomes [19]. These results are consistent with findings in other populations [48]. Potentially depression may be more associated with and a greater barrier to physical activity than anxiety, with further research needed.

In this sample, physic activity levels in women with PCOS were lower compared to active women without PCOS. Investigation into the barriers and motivators of physical activity in this study emphasised that despite having a medical condition and noting this as a motivator for 
physical activity, women with PCOS still noted time and fatigue as the key barriers to engaging in physical activity, similar to the control group. Having a positive attitude towards physical activity is insufficient for long-term physical activity engagement, as positive attitudes towards physical activity do not correspond with actual increases in physically active behaviour [49]. Additional factors such as addressing barriers and enhancing support are likely to be needed to increase engagement and sustainability in physical activity in PCOS. In this context, women with PCOS did note some specific barriers which should be considered in physical activity prescription. These included a lack of confidence in their ability to maintain physical activity, fear of injury and physical limitations. Given the role of physical activity in the management of PCOS, a lack of confidence in maintaining physical activity may indicate that women with PCOS need additional support and assistance in sustainable and effective physical activity programs. Physical limitations and fear of injury can be very inhibiting for an individual. Programs and advice may need to consider this barrier and develop strategies to give women with PCOS more confidence in their ability to perform physical activity safely and according to their physical abilities.

Women with and without PCOS noted similar motivators for physical activity. Women with PCOS were more likely to cite controlling a medical condition as a reason for doing physical activity compared to the control group, but only $40 \%$ of women with PCOS saw this as a motivator. No significant differences were observed for decreasing risk of disease or illness or controlling blood glucose levels as physical activity motivators between women with or without PCOS. This is despite the fact that women with PCOS have a higher mean BMI and greater risk of diabetes and cardio-metabolic risk factors [50]. This indicates that the importance of physical activity for managing current PCOS symptoms and minimising long-term complications of PCOS may not be fully understood by women with PCOS. As diet and physical activity are endorsed as the primary management strategies for PCOS in recent evidence based guidelines [26] better promotion of short and long term benefits of lifestyle modification is warranted to improve motivation for physical activity.

Women with PCOS indicated more support providers for physical activity than the control group which is positively associated with adherence to lifestyle modifications [49]. Whilst differences were observed across age groups in terms of children being support providers, this is likely to be due to the age of the women themselves and their children in the 18-30 years old group. Support from physical activity instructors and other health professionals could be a way to engage more women with physical activity for a longer time period [49]. This was especially pertinent for women with PCOS aged 18-
30 years, among whom one-third identified physical activity instructors and health professionals as important support providers or their physical activity pursuits.

Interestingly, when assessing the barriers, motivators and support providers for physical activity for women with and without PCOS with a healthy BMI, there were no differences between groups (data not shown). Whilst this does indicate that some of the barriers, motivators and support providers may be associated with the higher BMI, this difference may be relevant as women with PCOS are more likely to be overweight, and physical activity prescription should take this into consideration. However, despite reporting similar barriers, motivators and support providers, only $48 \%$ of women with PCOS were active compared to $69 \%$ of women without the condition $\left(X^{2}=0.050\right)$. Whilst standard scales and questionnaires can be used to measure barriers, motivators and support providers, there are clearly additional factors, unique to women with PCOS that are not being detected. In future research, investigation of barriers and motivators specifically related to PCOS would add value as it has been shown that hirsutism and acne can result in a negative self-image $[25,51]$ which is a significant barrier to physical activity [52]. Women with PCOS have also been found to sweat more than other women during physical activity [53]. The physical symptoms, in addition to an increased prevalence of obesity in this population have been found to be associated with an increased level of self-consciousness during physical activity [51]. The development of a tailored psychometrically validated questionnaire to examine the specific barriers and motivators of physical activity for women with PCOS is needed. Considering the geographic location, cultural environment and other unique population factors may also be important when administering questionnaires of this nature.

Limitations of this research include that it relies on selfreport, is cross-sectional in design, has uneven group numbers and has a limited measures of physical activity. Although both groups were recruited from the general community, more women with PCOS volunteered than women without PCOS and there were some demographic differences between groups with potential of volunteer bias leading to an under or over-estimation of effect. Also barriers and motivators to physical activity are assessed as frequencies rather than ranking and use a non-validated questionnaire with no available validated questionnaires in PCOS. Splitting the women into three age groups when assessing barriers, motivators and support providers does inherently reduce the variance associated with the measure. However, given the exploratory and hypothesis generating nature of the study, assessing barriers, motivators and support providers according to age assists in the planning of future research addressing the factors which help and/or hinder women with PCOS' physical activity plans. 
Strengths of the study include a large, multiethnic community-based sample, incorporating both anxiety and depression, and including a control group, which provides formative data on physical activity as a component of first line management in PCOS.

\section{Conclusions}

This study found that less women with PCOS were physically active compared to women without PCOS and that physical activity was associated with milder depression. While physical activity barriers, motivators and supports for women with PCOS are similar to those without PCOS, additional barriers in PCOS may limit engagement and sustainability of physical activity over the long term. This formative research will assist understanding of the interaction between mental health and active lifestyles for women with PCOS. Barriers, motivators and the need for support should be considered when prescribing and promoting of physical activity as a management strategy for PCOS. Future research may focus on eliciting specific physical activity barriers, motivators and support providers from women with PCOS in order to understand how to better prescribe physical activity as a management strategy to this population. Including theoretical frameworks in order to structure research may be advantageous, the development and use of validated questionnaires and of objective measures of physical activity may also strengthen findings in future research. Ultimately, current results support the need for more investigation regarding the physical and psychological impact of physical activity in PCOS to identify evidence-based strategies to optimise motivation for and engagement in sustainable physical activity.

\section{Abbreviations}

PCOS: Polycystic ovary syndrome; BMl: Body mass index;

TTM: Transtheoretical model; HADS: Hospital anxiety and depression scale; MANCOVA: Multivariate analysis of variance.

\section{Competing interests}

This project is supported by a BRIDGES Grant from the International Diabetes Federation. BRIDGES, an International Diabetes Federation project, is supported by an educational grant from Lilly Diabetes.

\section{Authors' contributions}

MGH \& HT were involved in the design of the study, acquisition of data and interpretation of results. NS was involved with design of the study and interpretation of results. LB and RP were involved in the analysis and interpretation. LB drafted the article and all authors contributed to the revisions and final version of the article. All authors have approved the final version of the article.

\section{Acknowledgements}

Dr Amanda Deeks participated in study design and study implementation. Ms Melanie Gibson-Helm is supported by an Australian Postgraduate Award. Professor Helena Teede is an NHMRC Research Fellow. A/Prof Nigel Stepto is supported through the Australian Government's Collaborative Research Networks (CRN) program.

\section{Author details}

${ }^{1}$ Institute of Sport Exercise and Active Living, Victoria University, Melbourne, Victoria, Australia. 'Women's Public Health Research, Monash Centre for
Health Research and Implenetation, School of Public Health and Preventive Medicine, Monash University, Clayton, Victoria, Australia. ${ }^{3}$ College of Sport and Exercise Science, Victoria University, PO Box 14428, Melbourne, Victoria

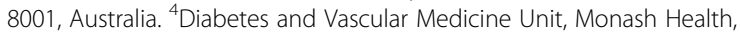

Clayton, Melbourne, Victoria, Australia.

Received: 28 November 2013 Accepted: 20 March 2014 Published: 27 March 2014

\section{References}

1. March WA, Moore VM, Wilson KJ, Phillips DI, Norman RJ, Davies MJ: The prevalence of polycystic ovary syndrome in a community sample assessed under contrasting diagnostic criteria. Hum Reprod 2010, 25:544-551.

2. Boyle J, Teede HJ: Polycystic ovary syndrome - an update. Aust Fam Physician 2012, 41(10):752-756.

3. Conway GS, Honour JW, Jacobs HS: Heterogeneity of the polycystic ovary syndrome: clinical, endocrine and ultrasound features in 556 patients. Clin Endocrinol 1989, 30:459-470.

4. Franks S: Polycystic ovary syndrome. N Engl J Med 1995, 333:853-861.

5. Jones GL, Balen AH, Ledger WL: Health-related quality of life in PCOS and related infertility: how can we assess this? Hum Fertil 2008, 11:173-185.

6. Moran $\mathrm{L}$, Teede $\mathrm{HJ}$ : Metabolic features of the reproductive phenotypes of polycystic ovary syndrome. Hum Reprod Update 2009, 15:477-488.

7. Teede HJ, Deeks AA, Moran L: Polycystic ovary syndrome: a complex condition with psychological, reproductive and metabolic manifestations that impacts on health across the lifespan. BMC Med 2010, 8:41.

8. Kiddy DS, Sharp PS, White DM, Scanlon MF, Mason HD, Bray CS, Polson DW, Reed MJ, Franks S: Differences in clinical and endocrine features between obese and non-obese subjects with polycystic ovary syndrome: an analysis of 263 consecutive cases. Clin Endocrinol 1990, 32(2):213-220.

9. Lim SS, Clifton PM, Noakes M, Norman RJ: Obesity management in women with polycystic ovary syndrome. Women Health (London, England) 2007, 3(1):73-86.

10. Dunaif A, Xinqi W, Lee A, Diamanti-Kandarakis E: Defects in insulin receptor signaling in vivo in the polycystic ovary syndrome (PCOS). Am J Physiol 2001, 281(2):E392.

11. Legro RS, Kunselman AR, Dunaif A: Prevalence and predictors of dyslipidemia in women with polycystic ovary syndrome. Am J Med 2001, 111(8):607-613.

12. Norman RJ, Masters L, Milner CR, Wang JX, Davies MJ: Relative risk of conversion from normoglycaemia to impaired glucose tolerance or non-insulin dependent diabetes mellitus in polycystic ovarian syndrome. Hum Reprod 2001, 16:1995-1998.

13. Stepto NK, Cassar S, Joham AE, Hutchison SK, Harrison CL, Goldstein RF, Teede HJ: Women with polycystic ovary syndrome have intrinsic insulin resistance on euglycaemic-hyperinsulaemic clamp. Hum Reprod 2013, 28:777-784.

14. Meyer C, McGrath BP, Teede HJ: Overweight women with polycystic ovary syndrome have evidence of subclinical cardiovascular disease. J Clin Endocrinol Metab 2005, 90(10):5711-5716.

15. Shaw LJ, Bairey Merz CN, Azziz R, Stanczyk FZ, Sopko G, Braunstein GD, Kelsey SF, Kip KE, Cooper-Dehoff RM, Johnson BD, Vaccarino V, Reis SE, Bittner V, Hodgson TK, Rogers W, Pepine CJ: Postmenopausal women with a history of irregular menses and elevated androgen measurements at high risk for worsening cardiovascular event-free survival: results from the National Institutes of Health-National Heart, Lung, and Blood Institute sponsored Women's Ischemia Syndrome Evaluation. J Clin Endocrinol Metab 2008, 93(4):1276-1284.

16. Barnard L, Ferriday D, Guenther N, Strauss B, Balen AH, Dye L: Quality of life and psychological well being in polycystic ovary syndrome. Hum Reprod 2007, 22:2279-2286.

17. Barry JA, Kuczmierczyk AR, Hardiman PJ: Anxiety and depression in polycystic ovary syndrome: a systematic review and meta-analysis. Hum Reprod 2011, 26(9):2442-2451.

18. Deeks AA, Gibson-Helm ME, Paul E, Teede HJ: Is having polycystic ovary syndrome a predictor of poor psychological function including anxiety and depression? Hum Reprod 2011. Epub 2011/03/26.

19. Moran LJ, Deeks AA, Gibson-Helm ME, Teede HJ: Psychological parameters in the reproductive phenotypes of polycystic ovary syndrome. Hum Reprod 2012, 27(7):2082-2088. 
20. Moran $\sqcup$, Gibson-Helm M, Teede HJ, Deeks AA: Polycystic ovary syndrome: a biopsychosocial understanding in young women to improve knowledge and treatment options. J Psychosom Obstet Gynecol 2010, 31(1):24-31.

21. Hedayati SS, Yalamanchili V, Finkelstein FO: A practical approach to the treatment of depression in patients with chronic kidney disease and end-stage renal disease. Kidney Int 2012, 81(3):247-255.

22. Lee H, Kim I, Lim Y, Jung HY, Park H-K: Depression and sleep disturbance in patients with chronic obstructive pulmonary disease. Geriatr Nurs 2011 32(6):408-417

23. Moussavi S, Chatterji S, Verdes E, Tandon A, Patel V, Ustun B: Depression, chronic diseases, and decrements in health: results from the World Health Surveys. Lancet 2007, 370(9590):851-858.

24. Wilhelm K, Mitchell P, Slade T, Brownhill S, Andrews G: Prevalence and correlates of DSM-IV major depression in an Australian national survey. J Affect Disord 2003, 75(2):155-162.

25. Kitzinger $C$, Willmott J: The thief of womanhood': women's experience of polycystic ovarian syndrome. Soc Sci Med (1982) 2002, 54(3):349-361.

26. Teede HJ, Misso ML, Deeks AA, Moran $\sqcup$, Stuckey BGA, Wong JLA, Norman RJ Costello MF: Assessment and management of polycystic ovary syndrome: summary of an evidence-based guideline. Med J Aus 2011, 195(6):S65-S112

27. Clark AM, Ledger W, Galletly C, Tomlinson L, Blaney F, Wang X, Norman RJ: Weight loss results in significant improvement in pregnancy and ovulation rates in anovulatory obese women. Hum Reprod 1995, 10(10):2705-2712.

28. Moran L, Harrison CL, Hutchison SK, Stepto NK, Strauss BJ, Teede HJ: Exercise decreases anti-müllerian hormone in anovulatory overweight women with polycystic ovary syndrome - a pilot study. Horm Metab Res 2011, 43(13):977-979.

29. Hutchison SK, Stepto NK, Harrison CL, Moran LJ, Strauss BJ, Teede HJ: Effects of exercise on insulin resistance and body composition in overweight and obese women with and without polycystic ovary syndrome. J Clin Endocrinol Metab 2011, 96(1):E48-E56.

30. Lamb JD, Johnstone EB, Rousseau J-A, Jones CL, Pasch LA, Cedars MI, Huddleston HG: Physical activity in women with polycystic ovary syndrome: prevalence, predictors, and positive health associations. Am J Obstet Gynecol 2011, 204(4):352.e351-352.e356.

31. Thomson RL, Buckley JD, Lim SS, Noakes M, Clifton PM, Norman RJ, Brinkworth GD: Lifestyle management improves quality of life and depression in overweight and obese women with polycystic ovary syndrome. Fertil Steril 2010, 94(5):1812-1816.

32. Carek PJ, Laibstain SE, Carek SM: Exercise for the treatment of depression and anxiety. Int J Psychiatry Med 2011, 41(1):15-28.

33. Eyre $H$, Baune BT: Neuroimmunological effects of physical exercise in depression. Brain Behav Immun 2012, 26(2):251-266.

34. Herring MP, Jacob ML, Suveg C, Dishman RK, O'Connor PJ: Feasibility of exercise training for the short-term treatment of generalized anxiety disorder: a randomized controlled trial. Psychother Psychosom 2011, 81(1):21-28.

35. Harrison $\mathrm{CL}$, Lombard CB, Moran $L$, Teede HJ: Exercise therapy in polycystic ovary syndrome: a systematic review. Hum Reprod Update 2011, 17(2):171-183.

36. Booth ML, Bauman A, Owen N, Gore CJ: Physical activity preferences, preferred sources of assistance, and perceived barriers to increased activity among physically inactive Australians. Prev Med 1997, 26(1):131-137.

37. The Rotterdam ESHRE/ASRM-Sponsored PCOS Consensus Workshop Group: Revised 2003 consensus on diagnostic criteria and long-term health risks related to polycystic ovary syndrome. Fertil Steril 2004, 81:19-25.

38. Mishra GD, Kuh D: Health symptoms during midlife in relation to menopausal transition: British prospective cohort study. BMJ: Brit Med J 2012, 344(7846):1-10.

39. Krul AJ, Daanen HAM, Hyegjoo C: Self-reported and measured weight, height and body mass index (BMI) in Italy, the Netherlands and North America. Eur J Pub Health 2011, 21(4):414-419.

40. Prochaska JO, DiClemente CC: Stages and processes of self-change of smoking: Toward an integrative model of change. J Consult Clin Psychol 1983, 51(3):390-395.

41. World Health Organization: Global Strategy for Diet, Physical Activity and Health. World Health Organization (Resolution WHA57.17); 2004.

42. Zigmond AS, Snaith RP: The hospital anxiety and depression scale. Acta Psychiatr Scand 1983, 67(6):361-370.
43. Moorey S, Greer S, Watson M, Gorman C, Rowden L, Tunmore R, Robertson B, Bliss J: The factor structure and factor stability of the hospital anxiety and depression scale in patients with cancer. Br J Psychiatry 1991, 158:255-259.

44. Australian Bureau of Statistics: Physical Activity: Main barriers and motivators report. In 4177.0 - Participation in Sports and Physical Recreation. Australia: Australian Bureau of Statistics; Canberra; 2007.

45. Wood BM, Nicholas MK, Blyth F, Asghari A, Gibson S: The utility of the short version of the depression anxiety stress scales (DASS-21) in elderly patients with persistent pain: does age make a difference? Pain Med 2010, 11(12):1780-1790.

46. Zhao G, Ford ES, Dhingra S, Li C, Strine TW, Mokdad AH: Depression and anxiety among US adults: associations with body mass index. Int J Obes 2009, 33(2):257-266

47. Harris-Glocker M, Davidson K, Kochman L, Guzick D, Hoeger K: Improvement in quality-of-life questionnaire measures in obese adolescent females with polycystic ovary syndrome treated with lifestyle changes and oral contraceptives, with or without metformin. Fertil Steril 2010, 93(3):1016-1019.

48. Midtgaard J, Stage M, Møøller T, Andersen C, Quist M, Røørth M, Herrstedt J, Vistisen K, Christiansen B, Adamsen L: Exercise may reduce depression but not anxiety in self-referred cancer patients undergoing chemotherapy. Post-hoc analysis of data from the "Body \& Cancer" trial. Acta Oncol 2011, 50(5):660-669.

49. Campbell K, Crawford D: Management of obesity: attitudes and practices of Australian dietitians. Int J Obes Relat Metab Disord 2000, 24(6):701.

50. Teede HJ, Joham AE, Paul E, Moran LJ, Loxton D, Jolley D, Lombard C: Longitudinal weight gain in women identified with polycystic ovary syndrome: results of an observational study in young women. Obesity (Silver Spring) 2013, 21(8):1526-1532.

51. Jones GL, Hall JM, Lashen HL, Balen AH, Ledger WL: Health-related quality of life among adolescents with polycystic ovary syndrome. $J$ Obstet Gynecol Neonatal Nurs: JOGNN / NAACOG 2011, 40(5):577-588.

52. Butt J, Weinberg RS, Breckon J, Claytor RR: Adolescent physical activity participation and motivational determinants across gender, age, and race. J Phys Act Health 2011, 8(8):1074-1083.

53. Stachenfeld NS, Yeckel CW, Taylor HS: Greater exercise sweating in obese women with polycystic ovary syndrome compared with obese controls. Med Sci Sports Exerc 2010, 42(9):1660-1668.

doi:10.1186/1472-6874-14-51

Cite this article as: Banting et al:: Physical activity and mental health in women with Polycystic Ovary Syndrome. BMC Women's Health 2014 14:51.

\section{Submit your next manuscript to BioMed Central and take full advantage of:}

- Convenient online submission

- Thorough peer review

- No space constraints or color figure charges

- Immediate publication on acceptance

- Inclusion in PubMed, CAS, Scopus and Google Scholar

- Research which is freely available for redistribution 